\title{
Prävalenz der latenten Tuberkulose-Infektion bei Beschäftigten im Gesundheitswesen - Ein Dreiländervergleich
}

\section{The Prevalence of Latent Tuberculosis Infections among Health-Care Workers - A Three-Country Comparison}

Autoren

Institute
A. Nienhaus ${ }^{1}$, A. Schablon ${ }^{1}$, D. Tripodi ${ }^{2}$, J. Torres Costa ${ }^{3}$

${ }^{1}$ Institut für Versorgungsforschung in der Dermatologie und bei Pflegeberufen (IVDP), Universitätsklinikum Eppendorf, Hamburg

${ }^{2}$ Abteilung für Arbeitsmedizin, Universitätsklinikum Nantes, Frankreich

${ }^{3}$ Abteilung für Arbeitsmedizin, Hospital São João, Alameda Professor Hernâni Monteiro, Porto, Portugal

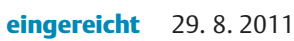
akzeptiert nach Revision 8. 9. 2011

\section{Bibliografie}

DOI http://dx.doi.org/

10.1055/s-0031-1291392

Online-Publikation: 17.10.2011

Pneumologie 2011; 65: 726-729

(c) Georg Thieme Verlag KG

Stuttgart · New York

ISSN 0934-8387

\section{Korrespondenzadresse}

Prof. Dr. med. Albert Nienhaus Institut für

Versorgungsforschung in der

Dermatologie und bei

Pflegeberufen

Universitätsklinikum Hamburg-

Eppendorf

Martinistraße 52

20246 Hamburg

a.nienhaus@uke.uni-

hamburg.de

\section{Zusammenfassung}

$\nabla$

Einleitung: Beschäftigte im Gesundheitswesen werden regelmäßig auf Tuberkulose untersucht. Systematische Auswertungen der Ergebnisse dieser Untersuchungen gibt es bisher aber nicht. Mit der Einführung der Interferon- $\gamma$ Release Assays (IGRA) wurde ein TB-Netz für Betriebsärzte aufgebaut, um die Erfahrungen mit dem IGRA bei den Vorsorgeuntersuchungen systematisch zu sammeln.

Methode: Bisher liegen die Daten von 2028 Vorsorgeuntersuchungen vor. Außerdem stehen die Daten von Vorsorgeuntersuchungen in Frankreich $(n=148)$ und Portugal $(n=2889)$ für eine kombinierte Analyse zur Verfügung. In den drei Kohorten wurde der QuantiFERON-TB ${ }^{\circledR}$ Gold in Tube sowie der Tuberkulin-Hauttest mit RT23 eingesetzt. Ergebnisse: Die Prävalenz der latenten Tuberkulose-Infektion (LTBI) ist abhängig vom Alter und vom Land. Von den jungen Beschäftigten $(<25$ Jahre) hatten in Deutschland 3\% und in Portugal $18 \%$ einen positiven IGRA. Bei über 55-jährigen Beschäftigten waren in Deutschland $25 \%$ und in Portugal $45 \%$ positiv. Im französischen Kollektiv war die Altersabhängigkeit nicht so eindeutig. Hier waren $23 \%$ der jungen Beschäftigten und $33 \%$ der über 55-jährigen Beschäftigten positiv. Eine aktive Tuberkulose wurde nur bei 12 Beschäftigten in Portugal diagnostiziert. Davon erkrankten vier innerhalb der folgenden beiden Jahre nach einem positiven IGRA. Das Progressionsrisiko nach einem positiven IGRA betrug $0,2 \%$ pro Jahr.

Diskussion: Bei jungen Beschäftigten im Gesundheitswesen ist die Prävalenz der LTBI gering. Ein positiver IGRA nach einem engen Kontakt zu einem infektiösen Patienten spricht hier für das Vorliegen einer frischen Infektion. Ansonsten scheinen alte Infektionen zu überwiegen, da es

\section{Abstract \\ $\nabla$}

Introduction: Health-care workers are regularly screened for tuberculosis. To date, there has been no systematic analysis of the results of such a screening. A TB network for company doctors was created when interferon- $\gamma$ release assays (IGRAs) were introduced in order to systematically collate their experience with IGRAs in preventive check-ups.

Method: Data have so far been recorded from 2,028 preventive check-ups. There are also data from preventive check-ups in France $(n=148)$ and Portugal $(n=2,889)$ for the purposes of a combined analysis. QuantiFERON-TB ${ }^{\circledR}$ Gold InTube and the tuberculin skin test with RT23 were used in the three cohorts.

Results: The prevalence of latent tuberculosis infections (LTBI) is dependent on age and country. Among young health-care workers $(<25$ years old), $3 \%$ had a positive IGRA in Germany, compared with $18 \%$ in Portugal. Among health-care workers aged 55 and over, $25 \%$ were positive in Germany and $45 \%$ were positive in Portugal. In the French cohort an increase from $23 \%$ to $33 \%$ was observed when the youngest and oldest age categories were compared. Active tuberculosis has so far been diagnosed in 12 health-care workers in Portugal, four of whom developed culturally confirmed TB within the first two years following a positive IGRA. The risk of progression subsequent to a positive IGRA was $0.2 \%$ per annum.

Discussion: There is a low prevalence of LTBI among young health-care workers. In them a positive IGRA following close contact with an infectious patient is likely to indicate recent infection. Apart from that, older infections appear to prevail, as the IGRA results depend greatly on age, and the risk of progression following a positive IGRA appears to be low in the study groups. 
eine starke Abhängigkeit der IGRA-Ergebnisse vom Alter gibt, und das Progressionsrisiko nach positivem IGRA scheint in den untersuchten Kollektiven eher gering zu sein. Die Indikation für eine Chemotherapie sollte daher zurückhaltend gestellt werden.

\section{Einleitung}

Trotz abnehmender Inzidenz der Tuberkulose (TB) in der Bevölkerung sind Beschäftigte im Gesundheitswesen (BiG) weiterhin infektionsgefährdet [1 - 3]. Daher ist es nach wie vor sinnvoll, exponierte BiG auf Tuberkulose zu untersuchen. Bis zum Jahr 2004 wurden die Vorsorgeuntersuchungen auf TB von den Betriebsärzten mit dem „Stempeltest“ durchgeführt. Da dieser Test vom Hersteller vom Markt genommen wurde, musste eine Umstellung auf den Tuberkulin-Hauttest (THT) nach Mendel-Mantoux erfolgen. Dieser Test fand in der betriebsärztlichen Praxis jedoch wenig Akzeptanz. Nach Fortbildungsveranstaltungen mit praktischen Übungen zur Applikation des THT, an denen etwa 300 Betriebsärzte teilnahmen, gab nur ein Viertel der Teilnehmer an, zukünftig den THT einsetzen zu wollen [4]. Nachdem die Interferon-Gamma Release Assays (IGRA) in Deutschland kommerziell verfügbar wurden, ist deshalb ab dem Jahr 2005 ein TB-Netz für Betriebsärzte aufgebaut worden, um die Nützlichkeit der IGRA bei Vorsorgeuntersuchungen auf TB bei Beschäftigten im Gesundheitswesen zu evaluieren. Die Ergebnisse dieser fortlaufenden Studie wurden wiederholt publiziert [5-11]. Die im Rahmen dieses Netzwerkes gesammelten Erfahrungen zur Prävalenz der latenten Tuberkulose-Infektion (LTBI) werden in dieser Übersichtsarbeit wiedergegeben. Ergänzt werden diese Erfahrungen durch Studien aus Frankreich und Portugal, für die die Autoren dieser Übersichtsarbeit die Datenauswertungen durchgeführt haben [12-14].

\section{Methoden}

Mittlerweile beteiligen sich 14 Krankenhäuser am TB-Netz und insgesamt 2028 BiG wurden bisher untersucht. Die Untersuchung der Beschäftigten erfolgt durch die zuständigen Betriebsärzte im Rahmen der Wahrnehmung ihrer Aufgaben nach dem Arbeitssicherheitsgesetz (ASiG) in Kombination mit der Verordnung zur arbeitsmedizinischen Vorsorge (ArbMedVV) [15]. Untersucht werden BiG von pneumologischen Stationen und von Laboren, die regelmäßig Kontakt zu infektiösen Patienten oder Materialien haben (Pflichtuntersuchungen) oder BiG, die ungeschützten, unvorhergesehen engen Kontakt zu infektiösen Patienten oder Materialien hatten (Angebotsuntersuchungen). Die Pflichtuntersuchungen werden jährlich wiederholt. Für die Studienzwecke werden folgende Daten in einem standardisierten Erhebungsbogen erfasst: Alter, Geschlecht, Geburtsland, TB-Anamnese, frühere THT, Untersuchungsanlass und quantitative IGRA-Ergebnisse. Die Bögen werden in anonymisierter Form an das Studienzentrum geschickt. Für den IGRA wird von der Berufsgenossenschaft für Gesundheitsdienst und Wohlfahrtpflege (BGW) im Rahmen dieser Studie ein Pauschalbetrag erstattet, sofern das Unternehmen bei der BGW versichert ist. Die übrigen Kosten für die arbeitsmedizinische Vorsorgeuntersuchung trägt der Arbeitgeber entsprechend seiner Aufgaben nach ASiG und ArbMedVV. Die Betriebsärzte des TB-Netzes verwenden überwiegend den QuantiFERON Gold In Tube (QFT), da dieser preisgünstiger ist als der TSPOT.TB und beide IGRA bei Screeninguntersuchungen auf das Vorliegen einer latenten TB-Infektion (LTBI) gleichwertig sind [16].
A positive IGRA should therefore be interpreted with caution as an indication of the need for chemotherapy.

Ergänzt wird das TB-Netz Betriebsärzte durch Kooperationen mit Betriebsärzten in Frankreich und Portugal. Am Universitätskrankenhaus in Nantes (Frankreich) wurden bei Umgebungsuntersuchungen 148 BiG mit dem QFT und dem THT untersucht [12]. Am Universitätskrankenhaus in Porto (Portugal) werden alle BiG entsprechend den Empfehlungen des Centre for Disease Control (CDC) auf TB untersucht $[13,14]$. Seit 2007 wurden für diese Vorsorgeuntersuchungen bei 2889 Beschäftigten der QFT und der THT gleichzeitig verwendet. In beiden Ländern wird für den THT das RT23 vom Serum Staten Institute in Kopenhagen (Dänemark) verwendet. Alle BiG aus diesen beiden Kollektiven hatten mindestens eine BCG-Impfung.

Alle drei Kohorten umfassen BiG mit wiederholtem Kontakt zu TB-Patienten oder infektiösen Materialien. Die Exposition wurde in allen drei Kohorten nur sehr grob erfasst und Zusammenhänge zwischen bestimmten Tätigkeiten oder anderen Expositionsmerkmalen und der Wahrscheinlichkeit einer LTBI wurde nicht gefunden. Eine Analyse der Wahrscheinlichkeit für eine frische LTBI aufgrund einer aktuellen Exposition ist deshalb nicht möglich. Daher wurden publizierte Daten aus Umgebungsuntersuchungen entsprechend dem Infektionsschutzgesetz (IfSG) verwendet [16], um den Anteil der frischen Infektionen in Abhängigkeit von der Dauer und Intensität der Exposition zu berechnen. Ausgehend von den für die einzelnen Expositionskategorien berechneten Odds Ratios (OR) wurde der Anteil frischer LTBI entsprechend folgender Formel berechnet: $(\mathrm{OR}-1) / \mathrm{OR}$.

\section{Ergebnisse}

Eine in der Kultur bestätigte TB wurde im Rahmen der Untersuchungen in Portugal bei 12 BiG beobachtet [14]. In der deutschen und in der französischen Studie wurde bisher keine aktive TB bei einem BiG festgestellt. Alle BiG mit aktiver TB waren QFT-positiv. Im THT waren 10 von 12 positiv (THT $>10 \mathrm{~mm}$ ). In Portugal entwickelten sich vier der beobachteten 12 aktiven TB-Erkrankungen innerhalb der folgenden beiden Jahre nach einem positiven QFT. Da 953 BiG einen positiven QFT hatten und nachverfolgt wurden, betrug die Progressionsrate pro Jahr 0,2\% [14].

Der Anteil von BiG mit positivem IGRA und gleichzeitig positivem THT schwankt in den drei Ländern zwischen 5,7\% und 30,5\% ( Tab.1). Der Anteil IGRA-negativer, THT-positiver BiG beträgt zwischen 18,4\% und 50\%. Der Anteil IGRA-positiver, THT-negativer $\mathrm{BiG}$ ist in allen drei Kohorten gering (2,1\% bis 3,8\%). Durch die Einführung der IGRA werden bei 40,2\% der kombinierten Kohorte Lungenübersichtsaufnahmen überflüssig, da die BiG zwar THT-positiv, aber IGRA-negativ sind. Der Anteil nicht interpretierbarer QFT-Ergebnisse betrug in den drei Kohorten unter 1\% (Daten nicht gezeigt).

Die Inzidenz der TB in der Allgemeinbevölkerung beträgt in Deutschland und Frankreich 5-6 Fälle pro 100000 Einwohner [11,12] und in Portugal 25 Fälle pro 100000 Einwohner [14]. Dementsprechend ist die Prävalenz eines positiven IGRA abhängig vom Alter der Beschäftigten sowie vom Land. Bei BiG unter 25 Jahren beträgt sie in Deutschland unter $2 \%$ und bei über 55-Jährigen 25\% ( Tab. 2). In Portugal ist die Prävalenz der LTBI ebenfalls abhängig vom Alter. Allerdings sind bereits 18,9\% der BiG, 
Tab. 1 Direkter Vergleich zwischen IGRA und THT bei Beschäftigten im Gesundheitswesen (BiG) in Portugal, Deutschland und Frankreich.

\begin{tabular}{|lccccc|} 
& Studienkollektiv & IGRA + /TST+ & IGRA-/TST+ & IGRA+/TST- & IGRA-/TST- \\
\hline Land & $\mathrm{N}$ & $\mathrm{N}(\%)$ & $\mathrm{N}(\%)$ & $\mathrm{N}(\%)$ & $\mathrm{N}(\%)$ \\
\hline Deutschland [6] & 261 & $15(5,7)$ & $48(18,4)$ & $10(3,8)$ & $188(72,4)$ \\
\hline Portugal [12] & 1218 & $371(30,5)$ & $532(43,7)$ & $26(2,1)$ & $289(23,7)$ \\
\hline Frankreich [11] & 148 & $23(15,5)$ & $74(50,0)$ & $5(3,3)$ & $46(31,1)$ \\
\hline gesamt & 1627 & $409(25,1)$ & $654(40,2)$ & $41(2,5)$ & $523(32,1)$ \\
\hline
\end{tabular}

Tab. 2 Rate positiver IGRA in Abhängigkeit von Alter und Land.

\begin{tabular}{|c|c|c|c|}
\hline & \multicolumn{3}{|l|}{ IGRA positiv } \\
\hline & $\begin{array}{l}\text { Deutschland } \\
(\mathrm{N}=2004) \\
{[10]}\end{array}$ & $\begin{array}{l}\text { Portugal } \\
(\mathrm{N}=2884) \\
{[13]}\end{array}$ & $\begin{array}{l}\text { Frankreich } \\
(\mathrm{N}=148) \\
{[11]}\end{array}$ \\
\hline Alter & $N(\%)$ & $\mathrm{N}(\%)$ & $\mathrm{N}(\%)$ \\
\hline$<25$ Jahre & $4(1,6)$ & $57(18,9)$ & $5(22,7)$ \\
\hline 25 - 35 Jahre & $32(6,7)$ & $364(28,3)$ & $5(14,7)$ \\
\hline $35-45$ Jahre & $47(8,2)$ & $223(36,1)$ & $7(16,3)$ \\
\hline 45-55 Jahre & $67(13,2)$ & $221(45,5)$ & $7(18,9)$ \\
\hline$>55$ Jahre & $48(25,0)$ & $88(45,1)$ & $4(33,3)$ \\
\hline gesamt & $218(10,9)$ & $953(33,0)$ & $28(18,9)$ \\
\hline
\end{tabular}

die jünger als 25 Jahre sind, infiziert. Die Prävalenz der LTBI steigt im Datensatz aus Frankreich ebenfalls mit dem Alter, dieser $\mathrm{Zu}-$ sammenhang ist aber nicht so eindeutig wie in Deutschland und Portugal. Bei Pflegeschülern ist der QFT nur selten positiv (2\%) und diejenigen, die positiv waren, hatten Risikofaktoren für eine LTBI (TB in der Familie, Migration aus einem Land mit hoher TBInzidenz) [10]. Es kann also für Deutschland davon ausgegangen werden, dass diejenigen, die eine Tätigkeit im Gesundheitswesen neu beginnen und keine Risikofaktoren für eine LTBI haben, nicht infiziert sind. Die Rate positiver IGRA bei Einstellungsuntersuchungen war in Portugal mit $28 \%$ deutlich höher. Allerdings hatte hier etwa ein Drittel der Beschäftigten bereits vor der Einstellungsuntersuchung im Gesundheitswesen gearbeitet [14]. Entsprechende Daten aus Frankreich sind nicht vorhanden.

Bei Kontaktpersonen aus der deutschen Allgemeinbevölkerung war die Wahrscheinlichkeit für positive IGRA abhängig von der Indexperson sowie von der Intensität und Dauer des Kontaktes [16]. Das höchste Risiko für einen positiven IGRA bestand bei lang anhaltendem (> 40 Stunden) Kontakt zu einem Sputum-positiven Indexfall $(\mathrm{OR}=18,6)$. Ein Kontakt $\mathrm{zu}$ einem hustenden, Sputum-positiven Indexfall war unabhängig von der Kontaktdauer ebenfalls ein starker Prädiktor für einen positiven IGRA (OR 10,6). Der Anteil frischer LTBI schwankte zwischen 52\% und $95 \%$.

\section{Diskussion}

Dieses ist die erste Arbeit, die einen Vergleich der Prävalenz der LTBI bei BiG in verschiedenen europäischen Ländern - Deutschland, Frankreich und Portugal - erlaubt. Entsprechend der Inzidenz der TB in den einzelnen Ländern unterscheidet sich auch die Prävalenz der LTBI bei den BiG. Der Anteil der BiG mit positivem THT, aber negativem IGRA ist in Frankreich und Portugal deutlich größer als in Deutschland. Das kann wahrscheinlich damit erklärt werden, dass in Frankreich und Portugal bei Beschäftigten im Gesundheitswesen eine BCG-Impfung durchgeführt wird, wenn der THT negativ ist. Für Frankreich gilt dies bis zum Jahr 2008 und für Portugal bis zum Jahr 2000. Dementsprechend waren $62 \%$ der französischen und $68 \%$ der portugiesischen $\mathrm{BiG}$ mehrmals BCG-geimpft [12,13]. In einer deutschen Metaanalyse konnte gezeigt werden, dass THT-positive, aber IGRA-negative Ergebnisse zu etwa $85 \%$ durch eine Kreuzreaktion mit einer BCG-Impfung erklärt werden [6].

Die Progressionsrate bei positivem IGRA scheint bei Beschäftigten im Gesundheitswesen deutlich geringer zu sein als bei engen Kontaktpersonen, die entsprechend des Infektionsschutzgesetzes (IfSG) im Rahmen von Umgebungsuntersuchungen getestet werden [16]. Kein BiG, der im Rahmen des TB-Netzes untersucht wurde und im QFT positiv war, entwickelte bisher eine aktive Tuberkulose. In Portugal entwickelten 4 von 953 BiG mit positivem QFT innerhalb der folgenden beiden Jahren eine aktive Tuberkulose (Progressionsrisiko pro Jahr 0,2\%) [14]. Im Vergleich dazu betrug die Progressionsrate bei engen Kontaktpersonen $12 \%$ für die ersten beiden Jahre nach Kontakt [15]. Drei Faktoren erklären wahrscheinlich das unterschiedliche Progressionsrisiko:

- Kinder haben ein höheres Progressionsrisiko als Erwachsene. Kinder arbeiten aber nicht im Gesundheitswesen [17].

- Das Progressionsrisiko ist am höchsten in den ersten beiden Jahren nach der Infektion. Bei Beschäftigten im Gesundheitswesen ist der Anteil derjenigen, die einen positiven IGRA aufgrund einer alten LTBI haben, wahrscheinlich höher als bei Umgebungsuntersuchungen in der Allgemeinbevölkerung. Bei engen Kontaktpersonen stieg die Rate positiver IGRA auf bis zu $58 \%$ je nach Kontaktsituation ( Tab. 3). Der Anteil

\begin{tabular}{|c|c|c|c|}
\hline \multirow[b]{2}{*}{ Mikroskopie-Index } & \multicolumn{3}{|c|}{ IGRA positiv } \\
\hline & N (\%) & OR (95\%Cl) & \% frische LTBI \\
\hline positiv & $154(36,5)$ & $2,1(1,5-2,9)$ & $52(33-66)$ \\
\hline enger Kontakt zu hustendem Index & $106(49,3)$ & $4,0(2,7-5,8)$ & $75(63-83)$ \\
\hline \multicolumn{4}{|l|}{ Kontaktzeit } \\
\hline$>=8-40 \mathrm{Std}$ & $31(19,9)$ & $1,8(1,0-3,2)$ & $75(0-69)$ \\
\hline$>40$ Std & $178(42,6)$ & $5,7(3,5-9,3)$ & $82(71-89)$ \\
\hline \multicolumn{4}{|l|}{ mikroskopisch positiver Index } \\
\hline und hustend & $76(58,5)$ & $10,6(6,2-18,1)$ & $91(84-94)$ \\
\hline und $>40$ Std Kontakt & $98(48,5)$ & $18,6(6,9-50,0)$ & $95(86-98)$ \\
\hline
\end{tabular}

Tab. 3 Anteil positiver IGRA und Odds Ratios (OR) bei 812 Kontaktpersonen in Abhängigkeit von der Infektiösität der Indexperson und der Art des Kontaktes [nach 14]. 
frischer LTBI lag in Abhängigkeit von verschiedenen Expositionskriterien zwischen 52\% und 95\% (nach [16]). Ähnliche Berechnungen sind für Beschäftigte im Gesundheitswesen mit den vorhandenen Daten nicht möglich. Die starke positive Assoziation zwischen dem Alter und der Rate positiver IGRA spricht aber dafür, dass der Anteil alter LTBI bei Beschäftigten im Gesundheitswesen hoch ist.

- Die typischen Risikofaktoren für eine TB, Armut und Alkoholismus [18], sind bei Beschäftigten im Gesundheitswesen wahrscheinlich weniger häufig als bei Kontaktpersonen.

Aufgrund des geringen Progressionsrisikos bei positivem IGRA im Rahmen von betriebsärztlichen Vorsorgeuntersuchungen ist es wahrscheinlich nicht angebracht, bei allen BiG mit einem positiven IGRA eine Chemoprävention durchzuführen. Bei jungen Beschäftigten im Gesundheitswesen, bei denen eine Vorsorgeuntersuchung aufgrund eines engen Kontaktes zu TB-Patienten durchgeführt wurde und bei denen frühere Kontakte nicht bekannt sind, kann davon ausgegangen werden, dass ein positiver IGRA eine frische LTBI anzeigt. Bei diesen BiG kann eine Chemoprävention sinnvoll sein.

Zusammenfassend wird durch die Einführung der IGRA bei den Vorsorgeuntersuchungen nach der ArbMedVV die Anzahl der Röntgenaufnahmen zum Ausschluss einer aktiven TB deutlich reduziert und dadurch die Untersuchung für die BiG angenehmer. Die relativ geringe Rate positiver IGRA sowie die Tatsache, dass bei 2028 Untersuchungen von BiG in Deutschland keine aktive TB gefunden wurde, bestätigen die starke Einschränkung der Anlässe für eine Vorsorgeuntersuchung entsprechend der Vorgaben der ArbMedVV. Die Daten sprechen ferner dafür, die Nachuntersuchungszeiten für Beschäftigte in der Pneumologie und im Labor mit bekanntem Kontakt zu infektiösen Patienten oder Materialien von bisher einem Jahr auf alle zwei oder drei Jahre zu erhöhen. Bei der Entscheidung über eine präventive Chemotherapie ist nicht nur das IGRA-Ergebnis relevant, sondern auch die Expositionssituation.

\section{Interessenkonflikt}

$\nabla$

Die Autoren geben an, das kein Interessenkonflikt besteht.

\section{Literatur}

1 Baussano I, Nunn P, Williams B et al. Tuberculosis among health care workers. Emerg Infect Dis 2011; 17: 488-494

2 De Vries G, Sebek MMGG, Lambregts-van Weezenbeck CSB. Healthcare workers with tuberculosis infected during work. Eur Respir J 2006; 28: $1216-1221$
3 Diel R, Seidler A, Nienhaus A et al. Occupational risk of tuberculosis transmission in a low incidence area. Respir Res 2005; 6: 35-45

4 Nienhaus A, Brandenburg S, Teschler H. Tuberkulose als Berufskrankheit - Ein Leitfaden zur Begutachtung und Vorsorge. Landsberg: Ecomed; 2009

5 Nienhaus A, Loddenkemper R, Hauer B et al. Latent tuberculosis infection in healthcare workers-evaluation of an Interferon-gamma release assay. Pneumologie 2007; 61: 219-223

6 Nienhaus A, Schablon A, Diel R. Interferon-Gamma Release Assay for the Diagnosis of Latent TB Infection - Analysis of Discordant Results, when Compared to the Tuberculin Skin Test. PLoS ONE 2008; 3: 2665

7 Nienhaus A, Schablon A, Le Bâcle C et al. Evaluation of the Interferongamma Release Assay in Healthcare Workers. Int Arch Occup Enviro Health 2008; 81: 295-300

8 Nienhaus A. Tuberkulose im Gesundheitswesen. Pneumologie 2009; 63: $23-30$

9 Schablon A, Beckmann G, Harling M et al. Prevalence of Latent Tuberculosis Infection among Health Care Workers in a hospital for pulmonary diseases. J Occup Med Toxicol 2009; 4: 1

10 Schablon A, Diner G, Anske U et al. Poster: Prävalenz der latenten Tuberkulose-Infektion bei Berufseinsteigern im Gesundheitswesen 51. Kongress der Deutschen Gesesllschaft für Pneumologie und Beatmungsmedizin e.V. 17. -23.3.2010. Hannover. Pneumologie 2010; 64 (Supplement 3): S233

11 Schablon A, Harling M, Diel R et al. Risk of latent TB infection in individuals employed in the healthcare sector in Germany: a multicentre prevalence study. BMC Infect Dis 2010; 10: 107

12 Tripodi D, Brunet-Court Nael V et al. Evaluation of the tuberculin skin test and the interferon-gamma release assay for TB screening in French healthcare workers. J Occup Med Toxicol 2009; 4: 30

13 Torres CJ, Sa R, Cardoso MJ et al. Tuberculosis screening in Portuguese healthcare workers using the tuberculin skin test and the interferongamma release assay. Eur Respir J 2009; 34: 1423-1428

14 Torres Costa J, Silva R et al. Screening for tuberculosis and prediction of disease in Portuguese healthcare workers. J Occup Med Toxicol 2011; 6: 19

15 Verordnung zur Rechtsvereinfachung und Stärkung der arbeitsmedizinischen Vorsorge vom Dezember 2008. Artikel 1: Verordnung zur arbeitsmedizinischen Vorsorge (ArbMedVV). Bundesgesetzblatt. 2008: $2768-2779$

16 Diel R, Loddenkemper R, Meywald-Walter K et al. Comparative Performance of Tuberculin Skin Test, QuantiFERON-TB-Gold In Tube Assay, and T-Spot TB Test in Contact Investigations for Tuberculosis. Chest 2009; 135: $1010-1018$

17 Diel R, Loddenkemper R, Niemann S et al. Negative and Positive Predictive Value of a Whole-Blood Interferon- $\gamma$ Release Assay for Developing Active Tuberculosis: An Update. Am J Respir Crit Care Med 2011; 183: $88-95$

18 Diel R, Schneider S, Meywald-Walter K et al. Epidemiology of tuberculosis in Hamburg, Germany - a longterm population-based analysis applying classical and molecular epidemiological techniques. J Clin Microbiol 2002; 40: $532-539$

Dieser Artikel wurde geändert gemäß folgendem Erratum vom 3. 8. 2012:

A. Nienhaus et al. Prävalenz der latenten Tuberkulose-Infektion bei Beschäftigten im Gesundheitswesen - Ein Dreiländervergleich. Pneumologie 2011; 65, 726-729

In der Autorenliste wurde der Name eines Co-Autors falsch wiedergegeben: Richtig muss es heißen: D. Tripodi. 